\title{
Erratum: Erratum to: Effectiveness of tapentadol prolonged release for the management of painful mucositis in head and neck cancers during intensity modulated radiation therapy
}

Rosario Mazzola $^{1}$ - Francesco Ricchetti ${ }^{1} \cdot$ Sergio Fersino ${ }^{1} \cdot$ Niccolò Giaj-Levra $^{1}$. Alba Fiorentino $^{1}$ - Maurizio Nicodemo ${ }^{2}$ - Sergio Albanese ${ }^{3} \cdot$ Stefania Gori $^{2}$. Filippo Alongi ${ }^{1}$

Published online: 2 August 2016

(C) Springer-Verlag Berlin Heidelberg 2016

Erratum to: Support Care Cancer

DOI: $10.1007 / \mathbf{s 0 0 5 2 0 - 0 1 6 - 3 3 5 1 - 7}$

The names of the authors were inadvertently inverted in the published paper and is now corrected in this article.

The online version of the original article can be found at http:10.1007 /s00520-016-3351-7.

Rosario Mazzola rosariomazzola@hotmail.it

1 Radiation Oncology, Sacro Cuore Don Calabria Cancer Care Center, Negrar-Verona, Italy

2 Medical Oncology, Sacro Cuore Don Calabria Cancer Care Center, Negrar-Verona, Italy

3 Head Neck Surgery, Sacro Cuore Don Calabria Cancer Care Center, Negrar-Verona, Italy 